\title{
The Impact of Work-Life Balance on the Wellbeing of Employees in the Telecom Sector
}

\author{
S. Vijaya Kumari ${ }^{1}$, Dr. A. Manor Selvi ${ }^{2}$ \\ ${ }^{1}$ Research Scholar, St.Peters University-Avadi \\ ${ }^{2}$ Assistant Professor, St.Peters University-Avadi
}

\begin{abstract}
Work-life balance is an important topic in both professional business practice and academic research. The literature shows that work-life balance is a central issue affecting wellbeing, as family and work are the most important elements of everyone's life. Any competing demands of work and family life cause conflict and negatively affect the wellbeing of workers. This study focused on assessing the impact of work-life balance determined by work-family conflict and family-work conflict on the wellbeing of individuals employed in the telecom sector employee in chennai. Wellbeing was measured by levels of family satisfaction, work satisfaction and psychological distress. Quantitative research methods were used for the study, and a sample population was chosen amongst participants who were single and in a relationship, female and male, with and without children, by using a convenient sampling method. The questionnaire used contained existing scales where the Cronbach's alpha coefficients were above the recommended 0.7 . Out of 190 distributed questionnaires, 114 were completed and returned, giving an overall returning rate of $60 \%$. The data was analyzed using IBM SPSS version 20.
\end{abstract}

Keywords: TELECOM

\section{How work-life balance impacts the wellbeing of employees in the telecom sector?}

The study identified the existence of negative effects of poor WLB determined by high levels of work-family conflict and family-work conflict on family satisfaction. This study also confirmed negative effects of poor WLB due to high levels of work-family conflict on work satisfaction and psychological health. Negative impacts of family-work conflict on work satisfaction and psychological health were not supported. This study also showed that the main causes of work-family conflicts were excessive working hours and inflexibility of work schedule. It was recognized that employers can improve WLB by implementing familyfriendly initiatives such as flexi-time, time off in lieu, compressed working week, childcare support and eldercare support.

\section{Work-Life Balance Overview}

Work-life balance is an important topic in both professional business practice and academic research. Work-life balance (WLB) has become a popular research area in different fields such as sociology (e.g. Allan, Loudoun \& Peetz, 2007), psychology (e.g. Greenhaus, 2008; Frone, 2000), human resource management (e.g. Grady, McCarthy, Darcy \& Kirrane, 2008; McDonald, Pini \& Bradley, 2007), organization studies (e.g. Kelly, Kossek, Hammer, Durham, Bray, Chermack, Murphy \& Kaskubar, 2008), and gender studies (e.g. Sullivan \& Smithson, 2007; Hill, 2005; Sullivan \& Lewis, 2001).

The majority of research on the correlation between work and family life refers to WLB and organisation policies, WLB and organisation culture, WLB and HR management, WLB and work commitment, WLB and absenteeism, WLB and gender equality, WLB and family life, and many more (Casper, Eby, Bordeaux, Lockwood \& Lambert, 2007).
There are a number of studies examining WLB and workers wellbeing. These studies however were mainly conducted in the United States (e.g. Grzywacz \& Marks, 2000), the United Kingdom (e.g. Wise, Bond \& Meikle, 2003), Australia and New Zealand (e.g. Bochner, 2003). In addition, several researchers (e.g. Greenhaus, Collins \& Shaw, 2003; and Grzywacz \& Marks, 2000) noticed that work and family literature, while assessing relationships between work and family domains, considers family life as time spent with a spouse and children, ignoring other important aspects of family, such as time spent with parents, siblings and other relatives.

This study refers to ,familyee in its traditional and nontraditional context, which includes married couples with children, as well as individuals of the opposite or the same sex, who are in partnered relationships, with or without children. Family in this study also refers to one's overall home-life, which apart from partners, spouses and children, also includes parents, siblings, and other relatives. The main aim of this paper is to examine the effects of WLB on the wellbeing of employees in telecom sector during hard economic times. Participants of this research were chosen amongst different employments in the telecom sector, and included male and female employees, single, in partnered relationships and married, with and without children.

Both work-family conflict and family-work conflict were examined in this study in order to assess individuals WLB levels. Aspects such as family satisfaction, work satisfaction and psychological health (determined by levels of psychological distress) were examined in order to assess the participants" wellbeing. 


\section{International Journal of Science and Research (IJSR) \\ ISSN (Online): 2319-7064}

Index Copernicus Value (2013): 6.14 | Impact Factor (2014): 5.611

\section{Review of Literature}

\section{Work-Life Balance Defined}

Work-life balance is vital for individualse wellbeing, organisations" performance and a functioning society (Grady et al., 2008). There are different beliefs on how work-life balance should be defined, measured and researched (Grzywacz \& Carlson, 2007). Different terms are also used by different researchers while referring to ,work-life balancee. For example, Frone (2003), Greenhaus et al. (2003) and Clark (2000) refer to the term, ,work-family balance"; Clarke et al. (2004) refer to ,work-family fitc; Burke (2000) refers to ,work-personal life balance“; and Grady et al. (2008) refer to „work-life balance“. As workfamily balance is often associated with traditional families, i.e., individuals who are married with children (Barnett \& Hyde, 2001), and this study refers to a family in both its traditional and non-traditional form; in order to therefore avoid any confusion, the term „,work-life balance ${ }^{\text {ec }}$ is used throughout this paper.

Grady et al. (2008, p.3) state that the term ,work-life balance ${ }^{e e}$ is more comprehensive and includes "family, community, recreation and personal time". As stated by Grady et al. (2008) WLB in its broad sense captures all aspects of employees" personal and work life; this suggests that WLB should be focused on individuals, families, workplaces, communities, and society as a whole. However, due to word count and time limits, this study excluded community and societal aspects, and focused on individuals, families and workplaces.

The literature indicates a number of WLB definitions. Clarke, Koch and Hill (2004, p.121) state that WLB is an "equilibrium or maintaining overall sense of harmony in life". Clark (2000, p.751) describes WLB as "satisfaction and good functioning at work and at home, with a minimum of role conflict". The focus on the domains of work and family is vital as family and work are regarded as the most important elements of everyone"s life, and any competing demands from work and family life cause conflict and negatively affect the wellbeing of workers (Clark, 2000; Frone, 2000).

Work-family research has generally been dominated by the study of family and work role interference. However, work and family role enhancement studies seem to be growing in recent years (Grzywacz \& Marks, 2000). Work and family role interference suggests that responsibilities in separate domains such as work and family compete with each other in terms of limited time, psychological resources and physical energy, which leads to negative outcomes in both areas (Greenhaus \& Beutell, 1985). In contrast, work and family role enhancement suggests that participation in multiple roles can lead to better functioning in other life domains (Barnett \& Rivers, 1996). This study focuses on work and family role interference, measured by work-family conflict and family-work conflict; however, study on work and family role enhancement is suggested for further research.

\section{Research Problem and Aim of the Research}

After reviewing literature pertaining to work and family balance, the author found that work-family conflict and family-work conflict play important roles in affecting WLB and wellbeing of employees. The author decided to use those two conflicts as the two main variables measuring WLB, and three variables- family satisfaction, work satisfaction and psychological distress- to measure employees" wellbeing. This study focuses on assessing how these variables relate to each other in order to establish the relationship between WLB and the wellbeing of telecom sector employees. Participants in this research were chosen from different organizations operating in telecom sector, and included male and female, married, in relationships and single employees, with and without children.

The literature indicates positive relationships between WLB and workers" wellbeing, and their outcomes affecting organizations performance. Moorman (1993, p.759) argues that "one of the most widely believed maxims of management is that a happy worker is a productive worker". This research should benefit individuals and organizations in terms of a deeper understanding of the importance of a healthy balance between work and family demands and their effects on people"s wellbeing and organizations"e performance. From employees" perspective, a better understanding of the importance of balancing work and family demands should help in recognizing the areas that negatively affect their wellbeing, and allow addressing these issues by seeking access to the family-friendly initiatives that would improve their work and family satisfaction and overall wellbeing. From an organization"s point of view, this paper should give managers better insights regarding connections between WLB and employees" wellbeing, and their impacts on employees" commitment, absenteeism, turnover, productivity and overall performance. This information may be useful to organizations in developing and implementing WLB policies. The following six hypotheses were developed for this study:

\section{Hypothesis 1}

It is proposed that work-family conflict will be negatively correlated with family satisfaction.

\section{Hypothesis 2}

It is proposed that work-family conflict will be negatively correlated with work satisfaction.

\section{Hypothesis 3}

It is proposed that work-family conflict will be positively correlated with psychological distress.

\section{Hypothesis 4}

It is proposed that family-work conflict will be negatively correlated with family satisfaction.

\section{Hypothesis 5}

It is proposed that family-work conflict will be negatively correlated with work satisfaction.

\section{Hypothesis 6}

It is proposed that family-work conflict will be positively correlated with psychological distress. 


\section{International Journal of Science and Research (IJSR) \\ ISSN (Online): 2319-7064}

Index Copernicus Value (2013): 6.14 | Impact Factor (2014): 5.611

\section{The Questionnaire}

This research was based on data collected from a self-report questionnaire made up of multiple questions. The questionnaire consists of five sections: 1) family-work conflict scale and 2) work-family conflict scale, which were used to measure participants "e work-life balance; 3) family satisfaction scale, 4) work satisfaction scale and 5) psychological health scale, which were used to asses participantse $^{\text {ee }}$ wellbeing. Individuals were also asked a variety of demographic questions, and were required to confirm the availability and the extent of use of the WLB initiatives in their organizations. The main objective of this research was to identify the key characteristics strongly related to the individuals ${ }^{\text {ee }}$ wellbeing in order to assess how WLB affects the wellbeing of participants.

\section{Practical Implications}

The results of this study show negative relationships between poor WLB and individuals ${ }^{\text {ee }}$ wellbeing, in particular negative impacts of work-family conflict on levels of work satisfaction, family satisfaction and psychological health. Lower levels of work satisfaction can lead to employees ${ }^{\text {ee }}$ higher dissatisfaction with the employer, lower commitments and productivity. Lower family satisfaction can influence work performance (Hill, 2005). Distress can result in decreased productivity and higher absenteeism (Layous et al., 2011), higher staff turnover, and poorer work quality (Seligman, 2011).

Organizations, managers and business owners should take into consideration the consequences of poor WLB stated above, as they impact business productivity and performance. Some employers may be reluctant to implement family-friendly initiatives due to costs; however, over the long term, implementing family-friendly initiatives may reduce costs of sick leave, staff turnover and low productivity, and be more favorable to the business in terms of costs, productivity and performance. It should be noted that WLB has been recognized by employees and employers as an important factor in achieving optimum wellbeing and job performance (Clark, 2001).

Findings from this study should help organizations, managers and business owners to recognize the importance of employees ${ }^{e e}$ wellbeing and job satisfaction, as these factors are closely connected to staff motivation, commitment and retention, which impact organizations ${ }^{\text {ee }}$ productivity and overall performance. The current study showed that individualse wellbeing benefits not only employees by way of higher work satisfaction, family satisfaction and better psychological health, but it also benefits employers by decreased absenteeism and turnover, increased motivation, productivity and performance (Grady et al., 2008; Burke, 2000). As extensive hours at work and a lack of work schedule flexibility were identified as the main causes of work-family conflict, employers can decrease work-family conflict by introducing flexi-time, time off in lieu, and compressed working week initiatives to their employees. Organizations providing such benefits seem to understand the relationship between greater WLB and retention of a competent workforce, and its effect on organizational commitment and profitability (Ryan \& Kossek, 2008).

These findings suggest that more employees may have childcare responsibilities and eldercare responsibilities, which can increase levels of work-family conflict (Frone et al., 1992; Greenhaus \& Beutell, 1985). In addition, this study recognized that in relation to childcare and eldercare support, over $98 \%$ of employers did not offer childcare support, and $93 \%$ did not offer eldercare support. Over 30\% of respondents needed but did not have childcare support and $30 \%$ needed but did not have eldercare support. These findings suggest that employers could reduce work-family conflict by introducing childcare and eldercare support. Organizations, managers and business owners should take those changing demographic characteristics into consideration in order to be able to develop and implement effective family-friendly initiatives, which would reduce work-family conflict, and improve WLB and the wellbeing of their employees.

\section{Findings of the Study}

\section{Descriptive Statistics}

The objective of this study was to assess the impact of WLB on the wellbeing of employees in the telecom sector employees. Work-family conflict and family-work conflict were used as the two main variables measuring WLB, and three variables- family satisfaction, work satisfaction and psychological distress- to measure employees ${ }^{e e}$ wellbeing. Work-family conflict and family-work conflict scales measured levels of interference between work and family lives, and high scores were indicative of high levels of conflict. Family satisfaction scale and work satisfaction scale assessed levels of participantse ${ }^{\text {ee }}$ satisfaction with their family lives and their work, and high scores were indicative of high levels of satisfaction. Psychological health scale measured appearance of distress, and high scores were indicative of high levels of participantse distress, therefore low levels of psychological health.

Six hypotheses were proposed and tested using correlation coefficients in order to examine associations between the variables. Prior to hypothesis testing, preliminary analyses were conducted in order to measure reliability of each variable, and to obtain the basic summary calculations in relation to the sample. These calculations included a mean and standard deviation for continuous variables, and frequencies for categorical variables. Descriptive statistics were also conducted to check for any violation of the assumptions underlying each test (Pallant, 2013).

Hypothesis 1 proposed that work-family conflict will be negatively correlated with family satisfaction. The relationship between work-family conflict (measured by work-family conflict scale) and family satisfaction (measured by family satisfaction scale) was examined using Pearson's product-moment correlation coefficient. There was a statistically significant medium negative correlation between the two variables, $\mathrm{r}=-.44, \mathrm{n}=111, \mathrm{p}<0.01$, which indicates that the more work-family conflict is experienced by individuals the less satisfied they are with their family lives. Therefore Hypothesis 1 was supported. 


\section{International Journal of Science and Research (IJSR) \\ ISSN (Online): 2319-7064 \\ Index Copernicus Value (2013): 6.14 | Impact Factor (2014): 5.611}

Hypothesis 2 proposed that work-family conflict will be negatively correlated with work satisfaction. The relationship between work-family conflict (measured by work-family conflict scale) and work satisfaction (measured by work satisfaction scale) was examined using Pearson "s product-moment correlation coefficient. There was a statistically significant but small negative correlation between the two variables, $\mathrm{r}=-.28, \mathrm{n}=113, \mathrm{p}<0.01$, which indicates that the more work-family conflict is experienced by individuals the less satisfied they are with their work. Therefore Hypothesis 2 was supported.

Hypothesis 3 proposed that work-family conflict will be positively correlated with psychological distress. The relationship between work-family conflict (measured by work-family conflict scale) and psychological distress (measured by psychological health scale) was examined using Pearson's product-moment correlation coefficient. There was a statistically significant and medium positive correlation between the two variables, $\mathrm{r}=.40, \mathrm{n}=113, \mathrm{p}<$ 0.01 , which indicates that the more work-family conflict is experienced by individuals the more psychological distress will be observed. Therefore Hypothesis 3 was supported.

Hypothesis 4 proposed that family-work conflict will be negatively correlated with family satisfaction. The relationship between family-work conflict (measured by family-work conflict scale) and family satisfaction (measured by family satisfaction scale) was examined using Pearson's product-moment correlation coefficient. There was a statistically significant but small negative correlation between the two variables, $\mathrm{r}=-.20, \mathrm{n}=112, \mathrm{p}<0.05$, which indicates that the more family-work conflict is experienced by individuals the less satisfied they are with their family lives. Therefore Hypothesis 4 was supported.

Hypothesis 5 proposed that family-work conflict will be negatively correlated with work satisfaction. The relationship between family-work conflict (measured by family-work conflict scale) and work satisfaction (measured by work satisfaction scale) was examined using Pearson's product-moment correlation coefficient. There was a small negative correlation between the two variables, $\mathrm{r}=-.15, \mathrm{n}=$ 113, which would indicate that the more family-work conflict is experienced by individuals the less satisfied they are with their work. However this correlation was not statistically significant $(\mathrm{p}>0.05)$, therefore Hypothesis 5 was not supported.

Hypothesis 6 proposed that family-work conflict will be positively correlated with psychological distress. The relationship between family-work conflict (measured by family-work conflict scale) and psychological distress (measured by psychological health scale) was examined using Pearsones product-moment correlation coefficient. However there was no statistically significant relationship between the family-work conflict and psychological distress, $\mathrm{r}=.03, \mathrm{n}=113, \mathrm{p}>0.05$. Therefore Hypothesis 6 was not supported. Results of all correlations are presented in Table
Table: Person's product-moment correlations between all variables

\begin{tabular}{|l|c|c|c|c|c|}
\hline Scale & $\mathbf{1}$ & $\mathbf{2}$ & $\mathbf{3}$ & $\mathbf{4}$ & $\mathbf{5}$ \\
\hline 1. Total work-family conflict - & & & & & \\
\hline 2. Total family-work conflict - & $48^{*}$ & - & & & \\
\hline 3. Total family satisfaction -. & $44^{*}$ & $-.20^{*}$ & - & & \\
\hline 4. Total work satisfaction - & $.28^{*}$ & -.15 & .50 & - & \\
\hline 5. Total psychological distress. & $40^{*}$ & .03 & $-.54^{* *}$ & $-.53^{* *}$ & - \\
\hline
\end{tabular}

$* \mathrm{p}<0.05$ (2-tailed)

$* * \mathrm{p}<0.01$ (2-tailed)

\section{Limitations}

Considering the various categories of employers in the private sector chosen for this study to gather data, and the sample size, it should be noted that the findings from this study are relevant to the sample population, and may not be generalisable to the entire employment in telecom sector. The findings may be relevant to similar occupations in similar businesses and organizations; however the results may not be relevant to the categories of employers not included in the sample. The size of the sample population (114 respondents) could also influence the statistical strength of this research. Therefore, future research on a larger sample population is recommended in order to increase the significance of the findings (Saunders et al., 2012). Also, future research should be conducted amongst different groups of employers using a bigger sample size, in order to obtain more information about different employment sectors in the private sector, which would lead to a better understanding of employment characteristics in the entire telecom sector employee.

This research was based on data collected from a self-report questionnaire made up of multiple questions. The results of the data collected may have been limited due to responses bias (Groves \& Peytcheva, 2008). Individuals who volunteered to take part in the research could have a stronger interest in the WLB topic than those who did not take part in the research. Also, an individual's circumstances while answering the questionnaire could have influenced the results. For example, if the questionnaire was completed at work, participants could have limited time allocated, and may not have read questions properly in order to fully understand what they were asked for. This could have lead to questions not being answered correctly. While completing questionnaires at work, answers may have been influenced by the presence of participants"e colleagues, managers or business owners. This could have prevented partakers from answering questions honestly, in particular questions asking about levels of work satisfaction, and how employers could improve WLB.

\section{Conclusion}

This study makes some contributions to WLB studies regarding telecom sector employees. The study identified the existence of negative effects of poor WLB due to high levels of work-family conflict and family-work conflict on family satisfaction. This study also confirmed negative effects of 


\section{International Journal of Science and Research (IJSR) \\ ISSN (Online): 2319-7064}

Index Copernicus Value (2013): 6.14 | Impact Factor (2014): 5.611

poor WLB due to high levels of work-family conflict on work satisfaction and psychological health. It was found that demands and pressure from work and family domains have an adverse impact on family satisfaction. In addition, higher levels of work demands and longer time spent in employment led to lower levels of quality time spent with families and lower family satisfaction. Work-family conflict resulted in lower satisfaction with work and commitment towards employers, and higher degrees of distress, concentration problems, sleeping problems, unhappiness, and lack of confidence.

This study did not confirm negative relationships between family-work conflict and work satisfaction, and family-work conflict and psychological health. Even though correlations between those variables would suggest a negative impact of high family-work conflict on work satisfaction and psychological health, these correlations did not reach statistical significance, and these arguments could not be supported.

This research showed that the main causes of work-family conflicts were excessive working hours and a lack of work schedule flexibility. The research also showed that apart from an increasing female workforce and two-income households, the telecom employee force is ageing. This could lead to increased demands for more flexible working arrangements for employees with eldercare and childcare responsibilities. It was recognized that employers can improve WLB by implementing family-friendly initiatives such as flexi-time, compressed working week, time off in lieu, childcare support and eldercare support. Findings from the current study are important to both employees and employers in terms of a deeper understanding of WLB and its effects on peoplees wellbeing, which consequently affects organizations ${ }^{\text {ee }}$ productivity and performance

\section{References}

[1] Punitha, Sangeetha Padmavathi (1999), „work life balance: their problems and constraints ${ }^{\text {ee }}$ Indian Journal of Labour Economics, Vol.42, No.4, pp.701-706.

[2] Pushpalatha (2013), ,A study on issues and challenges of women in work life balance with reference to telecom sector, International Journal of business and management, Vol.1, No.4, pp.42-48.

[3] Raghavulu, M.V. (2012), „Institutional Support for work life balance in India ${ }^{\text {ee }}$

[4] Raheem, A. Abdul and C. Prabhu (2007), ,work life balancee: Problems and Prospects. India ${ }^{\text {ee }}$ Economic empowerment through telecom sector. New Century Publications, New Delhi, India-2007.

[5] Raj Soshte (2009), „Empowerment and welfare of women in India ${ }^{e c}$, Journal of research in arts and education, Vol.1, No.2, pp.46-50. 